\title{
TNF-receptor-1 adaptor protein FAN mediates TNF-induced B16 melanoma motility and invasion
}

\author{
A Boecke ${ }^{1,6}$, A C Carstens ${ }^{1,6}$, C D Neacsu ${ }^{2}$, N Baschuk ${ }^{1,7}$, D Haubert ${ }^{1,3}$, H Kashkar ${ }^{1,4,5}$, O Utermöhlen ${ }^{1}$, \\ C Pongratz ${ }^{1}$ and $M$ Krönke A $^{*, 4,5}$
}

${ }^{1}$ Institute for Medical Microbiology, Immunology and Hygiene (IMMIH), University of Cologne, 50935 Köln, Germany; ${ }^{2}$ Institute for Biochemistry II, Joseph-Stelzmann-Str 52, 50931 Köln, Germany; ${ }^{3}$ Novartis Pharma AG, Biologics Process Research \& Development, CH-4002 Basel, Switzerland; ${ }^{4}$ Center for Molecular Medicine Cologne (CMMC), University of Cologne, 50935 Köln, Germany and ${ }^{5}$ Cologne Excellence Cluster on Cellular Stress Responses in Aging-Associated Diseases (CECAD), University of Cologne, 50935 Köln, Germany

Background: Locomotion of cancer cells can be induced by TNF and other motogenic factors secreted by cells of the tumour microenvironment such as macrophages. Based on our recent findings that the TNF receptor adaptor protein FAN mediates TNF-induced actin reorganisation and regulates the directed migration of immune cells responding to chemotactic cues, we addressed the role of FAN in cancer cell motility and the formation of invadopodia, a crucial feature in tumour invasion.

Methods: In B16 mouse melanoma cells, FAN was downregulated and the impact on FAN on cell motility and invasion was determined using in vitro assays and in vivo animal models.

Results: Like $\mathrm{FAN}^{-1-}$ murine embryonic fibroblasts, FAN-deficient B16 melanoma cells showed defective motility responses to TNF in vitro. In vivo FAN-deficient B16 melanoma cells produced significantly less disseminated tumours after i.v. injection into mice. Danio rerio used as a second in vivo model also revealed impaired spreading of FAN-deficient B16 melanoma cells. Furthermore, FAN mediated TNF-induced paxillin phosphorylation, metalloproteinase activation and increased extracellular matrix degradation, the hallmarks of functionally active invadopodia.

Conclusion: The results of our study suggest that FAN through promoting melanoma cellular motility and tumour invasiveness is critical for the tumour-promoting action of TNF.

It has become apparent that tumours are complex ecologies of different cell types and that the full manifestation of the malignant potential of transformed epithelial cells requires an appropriate support structure from the stroma. Haematopoietic cells are recruited to most tumours, and especially the tumour-associated macrophages can constitute a large portion of the tumour mass (Pollard, 2004). These macrophages have been implicated in the progression of cancer (Bingle et al, 2002; Balkwill et al, 2005). Signalling between macrophages and tumour cells affects bilaterally the activity of actin regulators such as WASP and N-WASP, resulting in the formation of podosomes in macrophages and invadopodia in tumour cells respectively (Yamaguchi et al, 2006), strongly suggesting that macrophages are obligate partners for tumour cell migration, invasion and metastasis (for review, see

\footnotetext{
*Correspondence: Dr M Krönke; E-mail: m.kroenke@uni-koeln.de

${ }^{6}$ These authors contributed equally to this work.

${ }^{7}$ Current address: Cancer Immunology Program, Peter MacCallum Cancer Centre, St. Andrews Place, East Melbourne, Victoria, 3002, Australia
}

Received 14 December 2012; revised 18 April 2013; accepted 19 April 2013; published online 14 May 2013

(C) 2013 Cancer Research UK. All rights reserved 0007 - 0920/13 
Condeelis and Pollard, 2006). The crucial feature of the tumour microenvironment is the cytokine-mediated communication between inflammatory and tumour cells. Tumor necrosis factor (TNF) is one of the key mediators of the tumour microenvironment that can be produced by both macrophages and tumour cells themselves and which exerts paradoxical tumour-suppressing as well as tumour-promoting activities (Balkwill, 2009). The tumour-promoting function of TNF in host-tumour interactions has been extensively elaborated and reviewed by Balkwill et al (2005).

The motility of cancer cells is driven by reorganisation of the cytoskeleton and of contacts between the cell and the extracellular matrix (ECM). This involves many of the same proteins that are required for normal cell motility, including members of the Rho family of small GTPases, actin-associated proteins and regulators (Ridley et al, 2003). Degradation of the ECM occurs via the concerted action of a number of proteases, including matrix metalloproteinases (MMPs), cathepsins and serine proteases such as seprase and uPA (Chang and Werb, 2001; Bauvois, 2004). Podosomes and invadopodia also contain proteins that are commonly found in focal adhesions, such as focal adhesion kinase (FAK), integrin receptors, paxillin, vinculin and Src (Linder and Aepfelbacher, 2003; Cervero et al, 2012). Several studies have shown that actin turnover is important in the formation of invadopodia in various invasive cancer cells (Yamaguchi and Condeelis, 2007). For example, a melanoma cell line required the invadopodial localisation of MT1-MMP for efficient ECM degradation (Nakahara et al, 1997). A genomic analysis of metastatic mouse B16 melanoma cells revealed enhanced expression of mainly two sets of genes that correlated with metastasis, which were involved in ECM assembly or in the regulation of the actin-based cytoskeleton, respectively (Clark et al, 2000). The mechanisms regulating the actin cytoskeleton in tumour cells are therefore of great interest in the context of tumour metastasis (Lai et al, 2008).

With regard to the possible impact of TNF on the actin cytoskeleton, Peppelenbosch et al (1999) suggested the involvement of the membrane proximal region of TNF-RI in TNFinduced actin polymerisation. Recently, we identified the WD-repeat protein FAN (factor associated with neutral sphingomyelinase activity) as a mediator of TNF-induced actin reorganisation and filopodia formation (Haubert et al, 2007). Specifically, FAN interacts with the actin cytoskeleton in TNFstimulated cells via direct $\mathrm{f}$-actin binding. It is an adaptor protein that binds to the $55 \mathrm{kD}$ receptor for TNF (TNF-RI) (Adam-Klages et al, 1996). It binds to the NSD of TNF-R1 (Adam et al, 1996) which is distinct of the death domain. The signalling targets of FAN include neutral sphingomyelinase, Rack1 and EED (Tcherkasowa et al, 2002; Philipp et al, 2010). Recent evidence suggested that TNF propels the motility of nonmalignant fibroblasts and leukocytes (Haubert et al, 2007; Montfort et al, 2009) as well as cancer cells (Rosen et al, 1991; Dekker et al, 1994; Hou et al, 2010). Yet the function of FAN in the context of TNF action remained ill defined. Montfort et al (2009) reported that FAN mediates TNF-induced expression of cytokine and chemokine genes and leukocyte recruitment in response to TNF. Furthermore, by using direct live imaging of translucent zebrafish larvae we could demonstrate in vivo that FAN is required for the directed chemotactic response of leukocytes to wounds and sites of infection (Boecke et al, 2012).

The role of FAN in TNF-induced actin reorganisation and leukocyte migration in zebrafish prompted us to address the question of whether FAN is possibly involved in tumour cell motility and metastasis. By employing genetic models of FANdeficient B16 melanoma cell lines and $\mathrm{FAN}^{-/-}$mice, we provide mechanistic insight into the role of FAN as a key mediator of TNF-induced melanoma cell motility and invasiveness.

\section{MATERIALS AND METHODS}

Cell lines. B16 F1 and F10 cell lines were purchased from ATCC (distributor LGC Promochem, Product codes ATCC-CRL-6475 and ATCC-CRL-6323, batch numbers 3794372 and F-12019). Mouse embryonic fibroblasts (MEFs) and B16F1 and F10 mouse melanoma cells were cultured at $37^{\circ} \mathrm{C}$ in DMEM and $10 \%$ fetal bovine serum (FBS). The HEK293FT cells were cultured as described supplemented with $2 \mathrm{~mm}$ L-glutamine, non-essential amino acids and $10 \mathrm{~mm}$ sodium pyruvate. The B16 shFAN and shScr cell lines were cultured like HEK cells plus $12 \mu \mathrm{g} \mathrm{ml}^{-1}$ (F1 cell lines) or $17 \mu \mathrm{g} \mathrm{ml}^{-1}$ (F10 cell lines) Blasticidin.

Transfection. Transient transfection of shRNA and pEGFP/FAN plasmid DNA into HEK293 cells was performed with Lipofectamin 2000 (Invitrogen, Darmstadt, Germany). For microscopy of FANGFP, B16 melanoma cells were transfected using a LONZA Nucleofector 2b Device (LONZA, Cologne, Germany) following the manufacturer's protocol.

Generation of stable FAN-deficient cell lines. For the generation of stable FAN-deficient melanoma cell lines, FAN-specific shRNAs were designed and cloned into vectors $\mathrm{pENTR} / \mathrm{siH} 1 / \mathrm{mDD}$-stuffer and GATEWAY-compatible lentiviral vector (Invitrogen) $\mathrm{pL}$ as described in Pongratz et al (2010). Viral particles were transduced using the ViraPower Lentiviral expression system according to the manufacturer's instructions (Invitrogen). Clones stably expressing the FAN shRNA were selected by fluorescence and blasticidin resistance and analysed by quantitative real-time PCR to assess the degree of FAN downregulation.

Motility analysis using time-lapse video microscopy. Cells were seeded on 12 -well plates $\mathrm{O} / \mathrm{N}$ and imaged at $37^{\circ} \mathrm{C}$ using a Leica inverted microscope DMIRE2 (Leica, Wetzlar, Germany) with $\mathrm{CO}_{2}$ supply and capture software FW4000. Phase contrast pictures were taken every $15 \mathrm{~min}$ over $10 \mathrm{~h}$, and movies were generated from the pictures using the microscope software. Migration paths of individual cells were tracked in the movies using DIAS Analysis software (Soll Technologies Inc., Iowa, IA, USA) and excellence RT software (Olympus, Hamburg, Germany). From the migration paths, total path length, net path length and overall speed were calculated using the DIAS software. For each condition, at least 12 cells were tracked in four independent experiments.

Western blotting. Samples were subjected to SDS-PAGE and blotted on nitrocellulose membranes (Whatman, VWR, Bruchsal, Germany). Membranes were blocked for $30 \mathrm{~min}$ and incubated with primary antibody at the appropriate dilution. After washing and incubation with secondary antibody for $1 \mathrm{~h}$, signals were detected on film (Amersham GE Healthcare, Buckinghamshire, UK) using ECL reagent (Amersham GE Lifescience, Freiburg, Germany). Antibodies used were anti-GFP (Roche Applied Science, Mannheim, Germany, clones 7.1 and 13.1, 1:1000) and anti-p-Paxillin (Santa Cruz Biotechnology, Heidelberg, Germany, sc-101774, $1: 200)$.

FACS analysis. Transfected HEK293FT cells were analysed for GFP expression using FACS Canto (BD, Heidelberg, Germany).

Animal experimentation. All animal experimentation have been carried out with local ethical committee approval and adhering to the guidelines of German jurisdiction and the guidelines for the welfare and use of animals in cancer research (Workman et al, 2010).

Subcutaneous implantation of tumour cells. Female C57 Bl/6 mice (CR), 6-8 weeks old, were shaved on the ventral side and injected s.c. into the flank with $4 \times 10^{5}$ cells of the following cell lines: B16F1 wt, B16F10 wt, B16 F1shFAN and B16 F10shFAN 
( $n=8$ for each cell line). The recipients were monitored daily for sign of illness. Tumour-bearing animals were killed when the tumours displayed severe ulceration for ethical reasons (Workman et al, 2010).

Intravenous injection of tumour cells. Female C57 Bl/6 mice (CR), 6-8 weeks old, were injected with $4 \times 10^{5}$ B16 F1, B16 F10, B16 F1shFAN or B16 F10shFAN in $300 \mu \mathrm{l}$ PBS i.v. into the tail vein. On the indicated days, the lungs were harvested and the number of macroscopically visible melanoma tumours on the surface of the lungs was counted (Workman et al, 2010).

B16 melanoma staining, zebrafish injection and incubations. B16 F10 wt melanoma cells and F10shFAN were stained with DiO (green fluorescence; Vybrant, Invitrogen). Cells were seeded in sixwell plates, grown to confluency and trypsinised. Subsequently, cells were washed with PBS, transferred to $1.5 \mathrm{ml}$ tubes and centrifuged for $5 \mathrm{~min}$ at $240 \mathrm{~g}$. Cells were re-suspended in PBS containing $\mathrm{DiO}\left(5 \mu \mathrm{ml}^{-1}\right)$. Cells were incubated for $20 \mathrm{~min}$ at $37^{\circ} \mathrm{C}$. After this period cells were centrifuged for $5 \mathrm{~min}$ at $240 \mathrm{~g}$, the supernatant discarded and the cells were washed twice with PBS. Cells were suspended in PBS for injection into the embryos. At 4 days post fertilisation (dpf), zebrafish embryos were anaesthetised with tricaine (Sigma, Hamburg, Germany).

A total of 100 cells were loaded into an injection needle (Hilgenberg, Malsfeld, Germany) and injected into the yolk of 4 dpf zebrafish embryos. After injection, embryos were incubated at $35^{\circ} \mathrm{C}$ for 2 days. Imaging was performed by transferring the embryos on a hollow grinding slide using a stereomicroscope setup (SMZ1500; DSFI1; Nikon, Düsseldorf, Germany) (Workman et al, 2010).

Immunofluorescence. Cells grown on coverslips were fixed with $3 \%$ paraformaldehyde/PBS for 20 min and blocked with 3\% BSA in PBS for $30 \mathrm{~min}$. Cells were permeabilised with $0.1 \%$ saponin during blocking. For f-actin staining, cells were incubated with AlexaFluor488- or AlexaFluor568-conjugated phalloidin (Invitrogen) in PBS/BSA/0.1\% saponin for $1 \mathrm{~h}$. For staining of MT1-MMP and Cortactin, slides were incubated overnight with the primary antibodies (Abcam, (EP1264Y) and (0.T.21), used 1:200) and for $1 \mathrm{~h}$ with secondary Alexa antibodies (Invitrogen, 1:500). Coverslips were mounted on glass slides and examined using an Olympus IX81 CellR fluorescence microscope or an Olympus FLUOVIEW FV1000 confocal microscope.

Paxillin phosphorylation. After stimulation with $100 \mathrm{ng} \mathrm{ml}^{-1}$ TNF, cells were lysed in TNE $(50 \mathrm{~mm}$ Tris- $\mathrm{HCl}, 100 \mathrm{~mm} \mathrm{NaCl}$, $0.1 \mathrm{~mm}$ EDTA, $1 \%$ NP-40, $10 \mathrm{~mm} \quad \beta$-glycerophosphate, $1 \mathrm{~mm}$ sodium orthovanadate, $\mathrm{pH}$ 7.4) and subjected to western blotting. The antibody used was anti-p-Paxillin (Santa Cruz Biotechnology, sc-101774, 1:200).

Degradation assay. Coverslips were coated with fluorescently labelled gelatine using fluorophoreAlexaFluor 594 Protein Labeling Kit (Invitrogen) according to the manufacturer's protocol. Sterile coverslips were put on a droplet containing $20 \mu \mathrm{l}$ AlexaFluor 594-gelatine. Excess gelatine was removed and the coated site of the coverslip was then put onto $100 \mu \mathrm{l}$ of $0.5 \%$ icecold glutaraldehyde/PBS solution and incubated for $15 \mathrm{~min}$ at $4{ }^{\circ} \mathrm{C}$. Coverslips were gently washed three times with PBS at RT followed by an incubation with sodium borohydride $\left(5 \mathrm{mg} \mathrm{ml}^{-1}\right.$ in PBS) for $3 \mathrm{~min}$ at RT. Coverslips were again washed with PBS and subsequently resterilised in $70 \%$ ethanol for $20 \mathrm{~min}$ at RT. After three additional washing steps in PBS, coverslips were quenched in serum-free medium for $1 \mathrm{~h}$ at $37^{\circ} \mathrm{C}$. The coating of coverslips resulted in a red fluorescent background. To assess the degradation of the ECM, B16 melanoma cells were grown on coverslips for $20 \mathrm{~h}$ in the presence or absence of $100 \mathrm{ng} \mathrm{ml}^{-1}$ TNF. Cells were fixed for 20 min with 3\% PFA and the black areas were evaluated as read-out for the degradation of the ECM using ImageJ software to determine the percentage of degraded area (http://rsbweb.nih.gov/ij).

MMP activity assay. To perform real-time continuous measurement of MMP activity, $5 \times 10^{4}$ B16 F10 melanoma cells were incubated at $37^{\circ} \mathrm{C}$ for $2 \mathrm{~h}$ in $100 \mu \mathrm{CO}_{2}$ independent media with $0.5 \mu \mathrm{M}$ fluorogenic MMP substrate (OmniMMP RED fluorogenic substrate, ENZO Lifescience, Lörrach, Germany). Increasing fluorescence indicated real-time enzyme activity. Fluorescence was measured at 5-min intervals with a Tecan Genios Pro Basic fluorescence reader (Tecan, Crailsheim, Germany). Emission wavelength was $590 \mathrm{~nm}$ and displayed as raw fluorescent units (RFUs). To quantify the differences between wt and F10shFAN melanoma cell lines, RFUs were plotted and the increase of fluorescence over time was determined (ratio of RFU/min). The ratio after TNF stimulation divided by the control value was used to calculate the induction of MMP activity.

Transwell migration assay. For the transwell migration assay, cells were treated following the manufacturer's protocol (CytoSelect 24-well cell invasion assay, collagen I, Cell Biolabs Inc., Biocat GmbH, Heidelberg, Germany). Numbers of cells that transmigrated through the matrix were evaluated with a cell counter.

Quantification of invadopodia formation. B16 mouse melanoma cells were seeded on glass coverslips, incubated $\mathrm{O} / \mathrm{N}$ and subsequently were stimulated with $100 \mathrm{ng} \mathrm{ml}^{-1} \mathrm{TNF}$ for $2 \mathrm{~h}$ or left untreated. Cells were stained for f-actin and cortactin and cells and invadopodia were defined as dot-like structures positive for f-actin and cortactin. Cells were counted (100 cells per slide).

Statistical analysis. Results were expressed as mean \pm s.e.m. Statistical analyses were standard two-tailed Student's $t$-test for two data sets using Prism (GraphPad Inc., La Jolla, CA, USA). The $P$-values of $<0.05\left(^{*}\right),<0.01\left(^{* *}\right)$ and $<0.001\left(^{* *}\right)$ were deemed as significant, highly significant, and most significant, respectively.

\section{RESULTS}

FAN deficiency decreases TNF-dependent cell motility. To address the general impact of FAN on cellular motility, we first used a genetic mouse model of FAN deficiency (Kreder et al, 1999). Mouse embryonic fibroblasts were prepared from wt and $\mathrm{FAN}^{-1-}$ mice and analysed for their respective spontaneous motility as well as their response to TNF. Using live cell imaging, the migration of FAN wt and FAN ${ }^{-1-}$ MEFs was monitored at 15-min intervals over $10 \mathrm{~h}$. The migratory paths of individual cells were tracked to visualise motility of the cells. When comparing the migratory paths of FAN wt and FAN ${ }^{-1-}$ cells in the absence of TNF, similar spontaneous migration was observed (Figure 1A). Treatment with TNF increased random migration in wt fibroblasts, whereas FAN $^{-1-}$ cells did not display enhanced motility. Specifically, total path length, net path length and overall speed increased in FAN wt MEFs after TNF stimulation but not in the FAN $^{-1-}$ cells (Figure 1B), indicating that FAN regulates cellular motility in response to TNF.

FAN mediates TNF-induced melanoma cell motility in vitro and is required in vivo for B16 melanoma invasion. To test whether FAN is also involved in the metastasis of tumour cells, we used two well-established B16 mouse melanoma cell lines, B16 F1 and B16 F10, representing melanoma cells with low and high metastatic potential, respectively. Stable FAN-deficient cell lines were generated using lentiviral shRNA expression system. To test if the generated shRNAs are functional, two different shRNAs were transiently co-transfected with pEGFP-C3/FAN into HEK293FT 

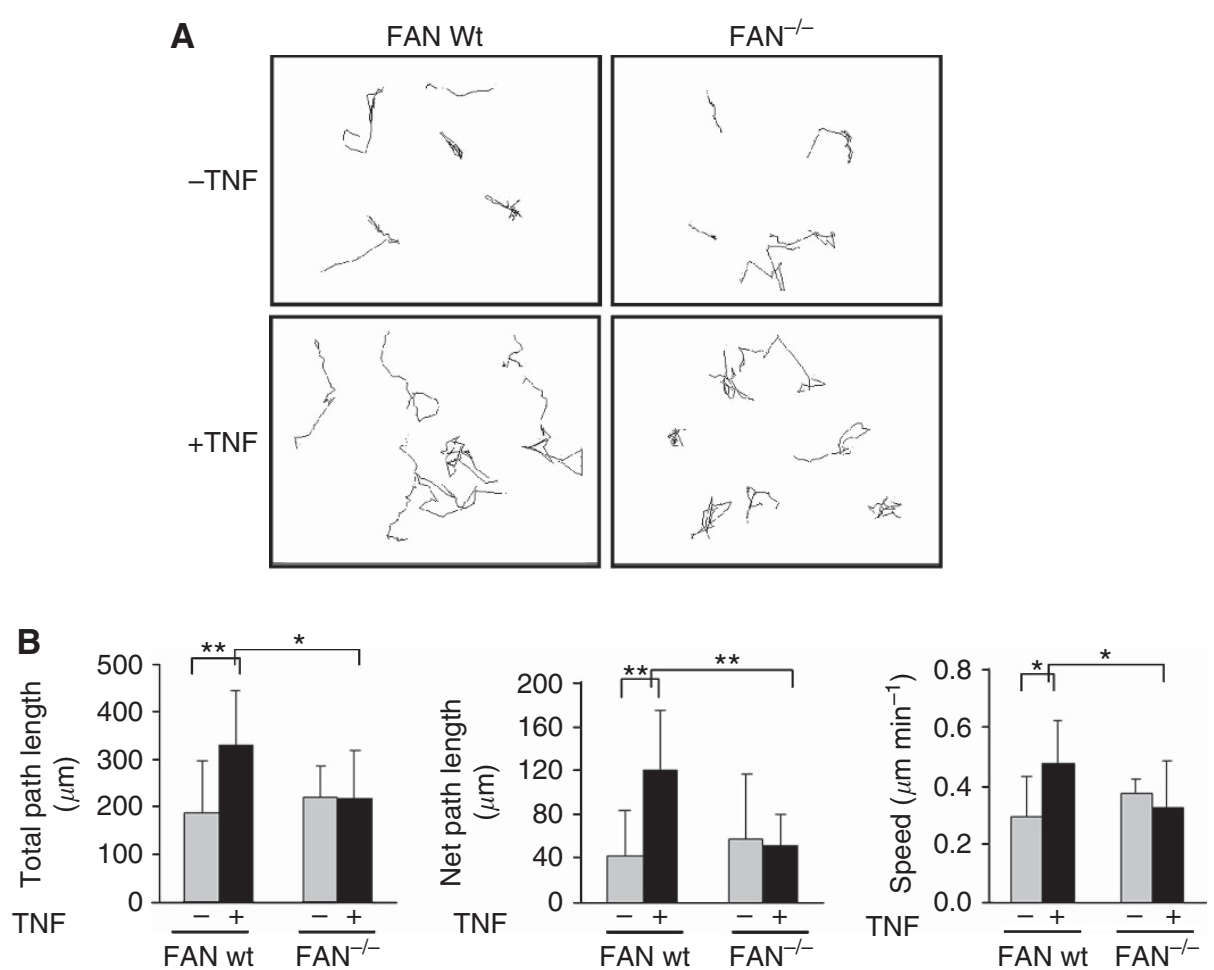

Figure 1. The deficiency of FAN decreases TNF-dependent MEF cell motility. (A) Representative migration tracks of individual FAN wt and FAN ${ }^{-1-}$ MEFs. Migratory behaviour of FAN wt and FAN ${ }^{-1}$ MEFs stimulated with TNF $\left(100 \mathrm{ng} \mathrm{ml}^{-1}\right)$ or left untreated. Images were taken every $15 \mathrm{~min}$ for $10 \mathrm{~h}$. (B) Quantification of migratory behaviour. Parameters from migration tracks were computed using DIAS software. Each bar represents the mean \pm s.d. of at least four independent experiments. Total path length represents the length of the track, whereas net path length is the distance between starting and end point of a track. Significance was measured using Student's t-test. ${ }^{*} P<0.05,{ }^{\star} * P<0.01$.

cells. Western blot analysis revealed that FAN-EGFP alone is clearly expressed and efficiently downregulated after co-transfection of FAN-specific shRNA (Figure 2A). The FACS analysis of transfected cells allowed quantification of the efficiency of the two different shRNAs (Figure 2B). Stable B16shFAN cell lines were generated using the functional shRNA and tested for FAN expression by quantitative RT-PCR. For each cell line, B16 F1 and F10, two clones were chosen that downregulate FAN to $\sim 60 \%$ (F1shFANC1) and $40 \%$ (F1shFANC2, Figure 2C) or $50 \%$ (F10shFANC1) and 30\% (F10shFANC2, Figure 2D). As control, we also generated stable lines expressing nontargeting scrambled shRNA (F1shScr and F10shScr). First, the possible impact of FAN deficiency on cell migration of B16 mouse melanoma cells was examined. No difference could be detected in the path length between different cell lines without TNF stimulation (Figure 2E and F). As for MEFs, the migration of B16 F1 control cells could be induced by TNF stimulation. Notably, the TNF-inducible migration was abrogated in F1shFAN and F10shFAN cells. Similar results were obtained with a transwell assay. As shown in Figure $2 \mathrm{G}$, the numbers of FAN-deficient cells migrating through the collagen matrix were markedly reduced in the presence and absence of TNF, providing a second line of evidence for the involvement of FAN in B16 melanoma migration. These concordant responses of two distinct parental melanoma cell lines and two respective independent FAN-deficient derivatives strongly argue against any cell line-specific effect.

FAN-deficient B16 mouse melanoma cells display reduced invasive activity in vivo. The impact of FAN on cellular motility prompted us to investigate the possible role of FAN in B16 melanoma cell migration and metastasis in vivo. First, B16 melanoma cells were implanted subcutaneously into wild-type mice
$(\mathrm{C} 57 \mathrm{BL} / 6)$ and the survival rate of these mice was determined. Mice challenged with B16 F1 wt cells died within 24 days, whereas the B16 F1 shFAN produced $50 \%$ lethality (Figure $3 \mathrm{~A}$ ). Mice challenged with B16 F10 wt cells died within 27 days. At this point, $40 \%$ of the mice that had been implanted with the B16 F10 shFAN cells were still alive. Taken together, mice that had been challenged with FANdeficient B16 mouse melanoma exhibited higher survival rates.

To address the question of whether FAN-deficient B16 mouse melanoma cells also display a reduction of disseminated tumours, B16 wt, F1shFAN or F10shFAN mouse melanoma cells were injected into the tail vein of wild-type mice. The lungs of injected mice were dissected 19 days after injection and the number of distinct lung tumours was determined (Figure 3B). The B16 F1 wt but not F1shFAN cells produced tumours in the lungs. The B16 F10 wt cells produced larger numbers of lung tumours. Notably, even these highly aggressive B16 F10 mouse melanoma cells did not form tumours when FAN is downregulated.

To support the notion that FAN is essentially involved in tumour cell migration, fluorescently labelled B16 F10 wt or shFAN cells were injected into the yolk sac of 4 dpf zebrafish embryos. After 2 days, imaging of zebrafish embryos was performed to visualise the melanoma cells that emigrated from the yolk sac and disseminated into the host's body. The number of FAN-deficient B16 melanoma cells found outside of the yolk sac was significantly reduced (Figure 3C and D). Strikingly, the number of embryos surviving the challenge with FAN-deficient B16 melanoma cells was significantly larger (Figure $3 \mathrm{E}$ ). Taken together, two independent in vivo models show that FAN is crucially involved in tumour cell systemic dissemination and migration.

Role of FAN in invadopodia formation. The dissemination and metastasis of cancer cells is associated with the formation of 

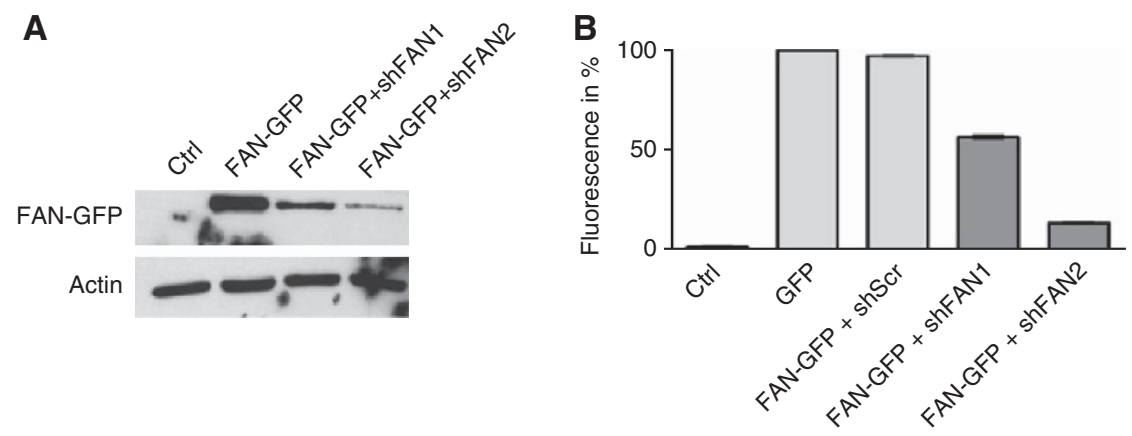
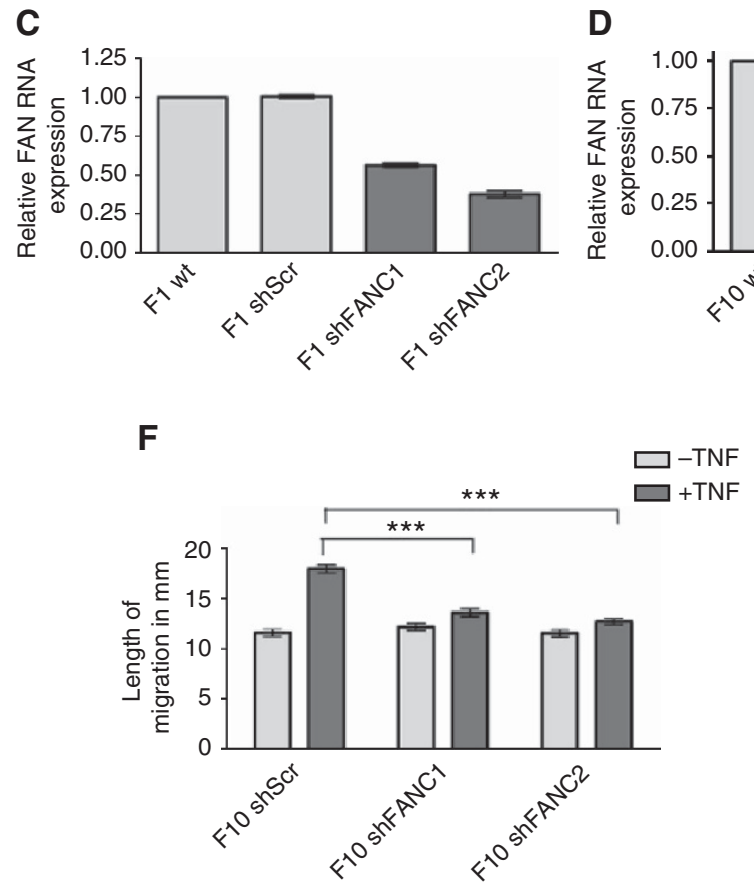
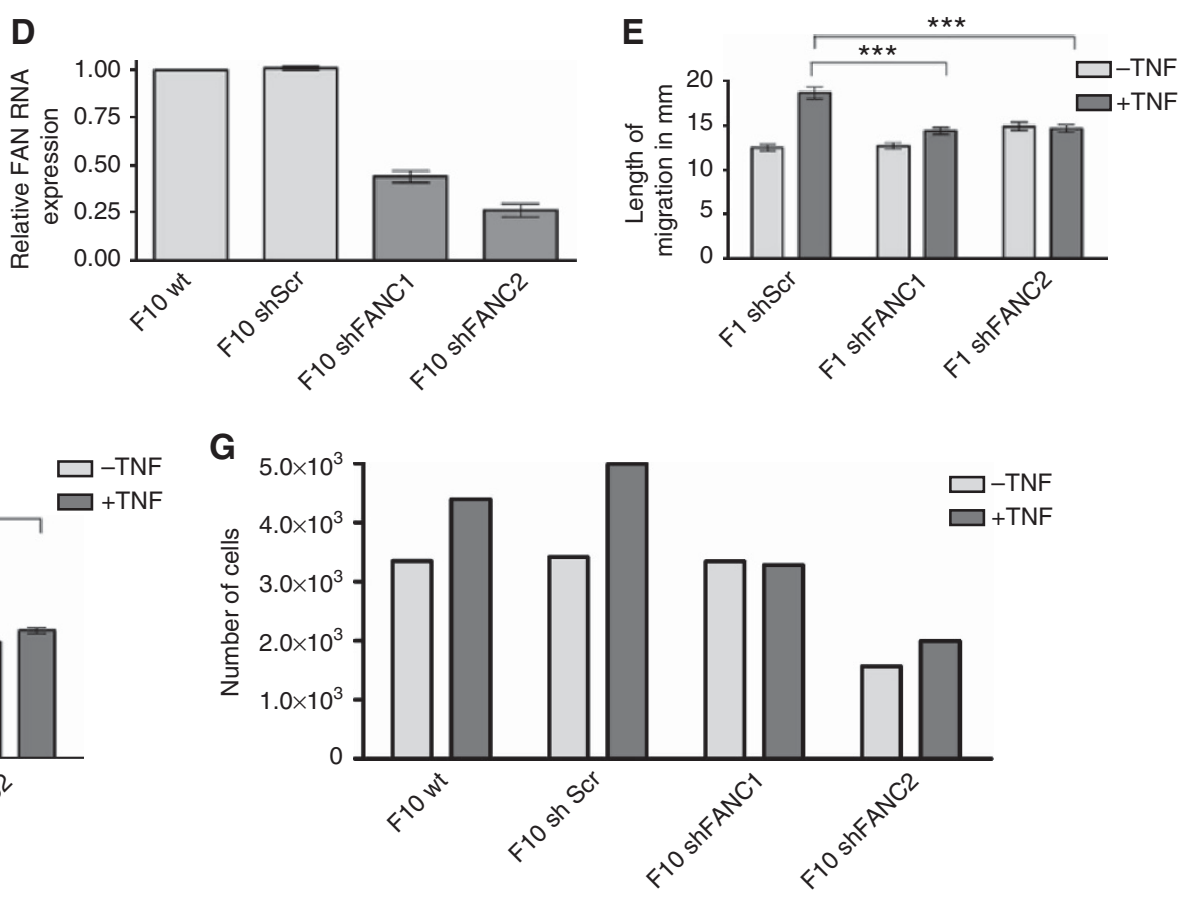

Figure 2. Tumor necrosis factor (TNF)-induced cell motility of B16 melanoma cells. (A) Targeting of FAN by FAN-specific shRNA constructs. The HEK293FT cells were either mock transfected or transfected with pEGFP-C3/FAN alone or in combination with one of the two FANshRNA constructs. Cell lysates were prepared, separated by SDS-PAGE and analysed by western blotting using anti-GFP antibody. (B) HEK293FT cells were either mock transfected or transfected with pEGFP-C3 or transfected with PEGFP-C3/FAN alone or in combination with one of the two pENTR/siH1/FANshRNA constructs. Cells were analysed by flow cytometry. (C and D) Quantitative RT-PCR to measure the relative FAN mRNA expression in B16 melanoma cell lines. Two clones for each of B16F1 and B16F10 that stably downregulate FAN were chosen for further experiments. (E and F) Migration in B16 F1 and F10 melanoma cell lines after TNF stimulation. Migration was measured every 15 min for $10 \mathrm{~h}$. Of each cell line, 100 cells were analysed using excellence RT software (Olympus). Results are expressed as mean \pm s.e.m. Statistical analyses were standard two-tailed Student's t-test for two data sets using Prism (GraphPad Inc.). The P-values of $<0.05\left(^{\star}\right)$, $<0.01\left(^{(\star *}\right)$ and $<0.001\left(^{\star \star \star}\right)$ were deemed as significant, highly significant, and most significant, respectively. (G) Transwell migration assay of B16 F10 melanoma cells. $1.25 \times 10^{5}$ cells were seeded on a collagen matrix. Cells that passed through the matrix were counted after $24 \mathrm{~h}$ using a Beckman Coulter cell counter, Krefeld, Germany.

invadopodia, that is, protrusions emanating from the surface of cancer cells exhibiting a very high proteolytic activity towards the ECM (Mueller and Chen, 1991; Murphy and Courtneidge, 2011). Indeed, immunofluorescence analysis of B16 melanoma cells stained for cortactin and f-actin revealed the presence of invadopodia (Figure 4A). The presence of FAN in invadopodia was revealed by colocalisation of FAN-GFP with $\mathrm{f}$ - actin, cortactin and membrane type1-MMP (MT1-MMP) (Figure 4B-D), suggesting that FAN interacts with core structures of invadopodia (Artym et al, 2006). The FAN deficiency resulted in a decreased number of cells displaying invadopodia in the presence or absence of TNF (Supplementary Figure S1). The most pronounced differences were observed with F10shFANC2, the B16 cell line showing most effective FAN downregulation (Figure 2A). The spatiotemporal organisation of invadopodia, and especially invadopodia disassembly, is controlled by paxillin phosphorylation (Badowski et al, 2008). As shown in Supplementary Figure S2, TNF induces phosphorylation of paxillin in wt MEFs and B16 melanoma cells, which was abrogated in FAN-deficient cells. The essential role of FAN for TNF-induced paxillin phosphorylation suggests that FAN is involved in the dynamics of invadopodia formation and disassembly.

The degradation of the ECM is reduced in FAN-deficient B16 melanoma cells. Tumour invasiveness mediated by invadopodia relies on their ability to degrade the ECM (Bowden et al, 1999). The degradation of ECM is prerequisite for cancer cells to evade the primary tumour site and to invade tissues for settling down in distinct environments. We therefore characterised the degrading activity of wt and FAN-deficient B16 melanoma cells towards the 
A

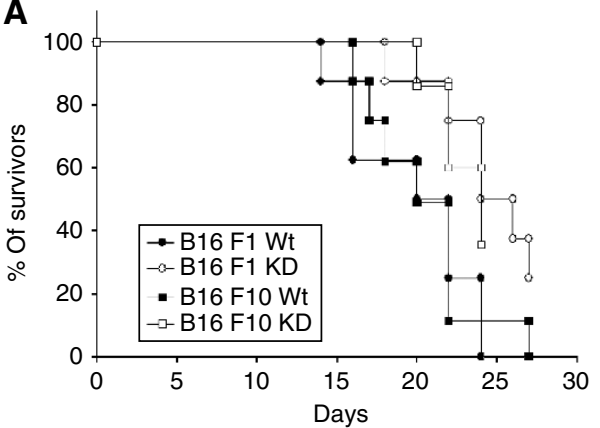

C
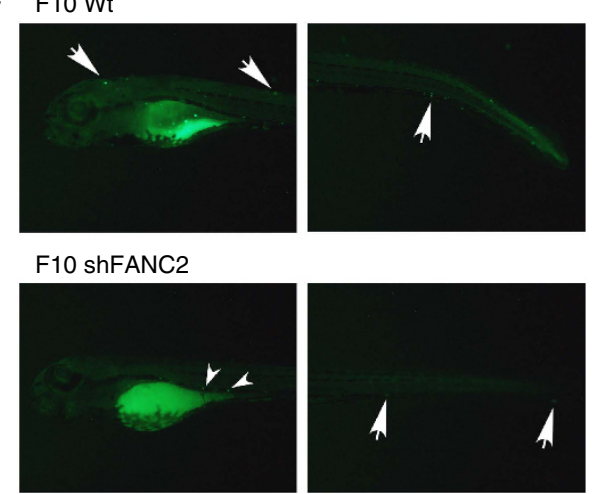

D
B

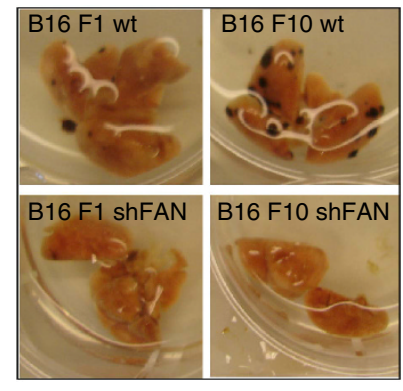

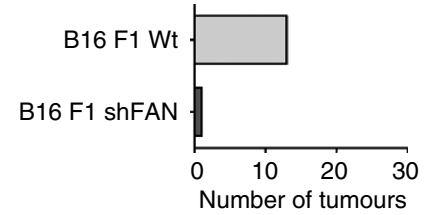

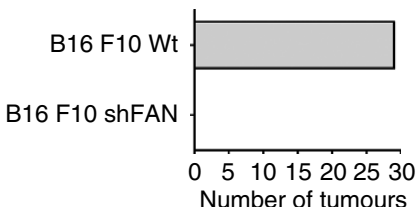

E
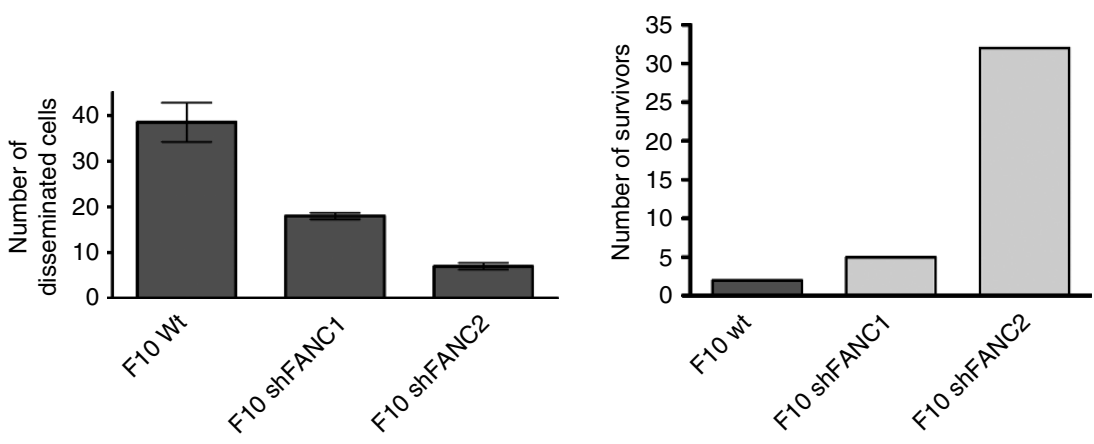

Figure 3. The FAN-deficient B16 cells display reduced invasive activity in vivo. (A) Survival of wild-type mice after subcutaneous (s.c.) injection with B16 mouse melanoma cells. (B) Dissected lungs of mice injected with wt and FAN-deficient B16 melanoma cells $(n=6)$. On day 16 , the lungs were harvested and the number of macroscopically visible tumours on the surface of the lungs was counted independently by two investigators. (C) Zebrafish were injected in the yolk sac with either F10 wt cells or F10shFANC2 cells. Arrows indicate disseminated tumour cells. (D) Quantification of cell dissemination. The number of cells that disseminated into the body were counted independently by two investigators ( $n=5$ fish per cell line). Results are expressed as mean \pm s.e.m. (E) Survival of zebrafish embryos after tumour cell injection. Zebrafish embryos injected with the FAN-deficient cell lines display a higher survival rate compared with embryos injected with wt cells 48 hpi (hours post injection).

ECM. The B16 F10 wt and F10shScr cells plated on red fluorescent gelatine resulted in loss of fluorescence after $20 \mathrm{~h}$ (Figure 5A), indicative of the degradation of the ECM. In contrast, F10shFANC1 displayed significantly less matrix degradation, which was also observed with F10shFANC2. The extent of matrix degradation was determined for each cell line by using ImageJ software, revealing a statistically significant loss of matrixdegrading activity in FAN-deficient B16F10 cell lines (Figure 5B). To investigate whether FAN-GFP colocalises with cortactin at sites of degradation, we analysed stacks obtained by widefield epi-fluorescence microscopy. As shown in Supplementary Figure S3A, FAN-GFP colocalised with cortactin in B16 melanoma cells contacting the matrix. Sharp images of FANGFP/cortactin puncta were only observed at the focal plane of the matrix (Supplementary Figure S3B) and blurred with increasing distance from the focal plane of the matrix, indicating that FAN-GFP localises to the ventral membrane in proximity of the matrix.

Tumor necrosis factor enhanced the degrading activity of F10 wt cells (Figure 6A and B). In contrast, this TNF response was almost completely abrogated in FAN-deficient B16 melanoma cells (Figure 6A and $\mathrm{B}$ ), indicative of the essential role of FAN in the spontaneous and inducible ECM-degrading activity of B16 melanoma cells.

FAN mediates TNF-induced metalloproteinase activation. The marked proteolytic activity of invadopodia towards the ECM is conferred by members of the metalloproteinase and serine proteinase families (Linder, 2007). The role of FAN in activation of metalloproteinases was assessed by a real-time MMP activity assay. The B16 melanoma cells incubated with a fluorogenic substrate emitted an increasing fluorescent signal indicating MMP activity (Figure 7A). The spontaneous metalloproteinase activity of wt and FAN-deficient B16 melanoma cells was almost indistinguishable (Figure 7B). Correspondingly, FAN-deficient B16 melanoma cells expressed similar levels of both pro- and active forms of MT1-MMP protein (Figure 7D). In contrast, wt cells showed enhanced MMP activity when stimulated with TNF, whereas FAN-deficient B16 melanoma cells did not significantly respond to TNF stimulation (Figure $7 \mathrm{~B}$ and $\mathrm{C}$ ), suggesting that FAN mediates TNF-induced enhancement of metalloproteinase activity in invadopodia.

\section{DISCUSSION}

Inflammatory cells are components of the tumour microenvironment that exert pro- as well as anti-tumoural activity. The crucial feature of the tumour microenvironment is the cytokine-mediated communication between the inflammatory and tumour cells. Tumor necrosis factor is one of the key mediators of the tumour microenvironment (Balkwill, 2009) that links inflammation to tumour motility and metastasis. In particular, it has been reported that TNF stimulates the motility of epithelial tumour cells (Rosen et al, 1991) including melanoma cells (Zhu et al, 2004). However, the molecular mechanisms of TNF action underlying the functional link to cell migration and invasiveness remained ill-defined. By using FAN-deficient B16 melanoma cells and two independent in vivo host models, we here demonstrate the essential 
A

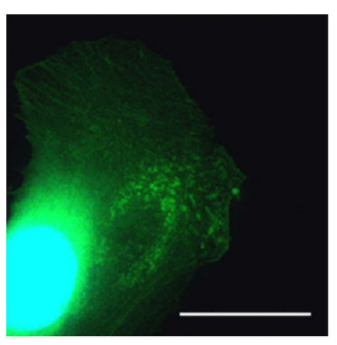

B

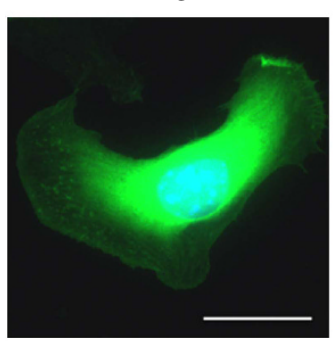

C FAN-GFP

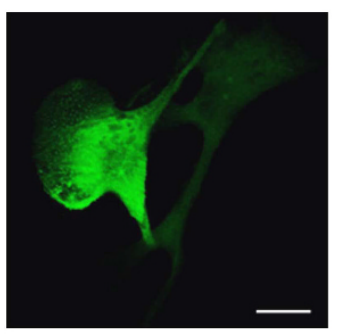

D

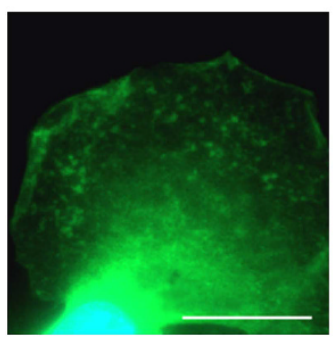

Cortactin

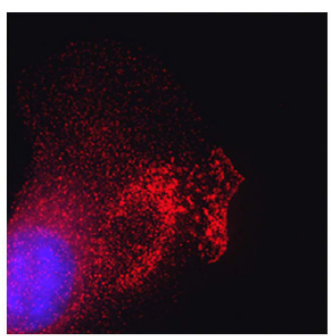

Phalloidin

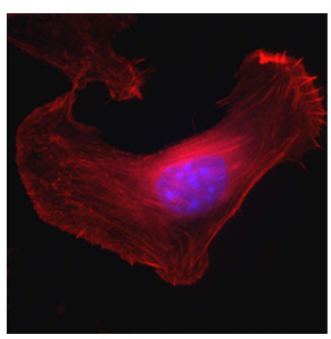

Cortactin

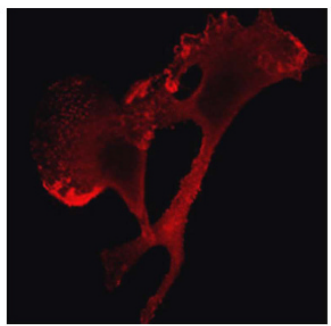

MT1-MMP

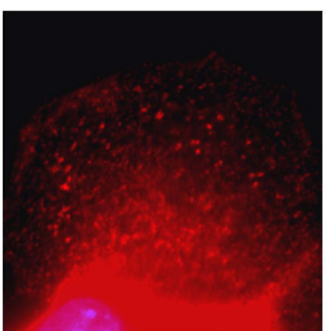

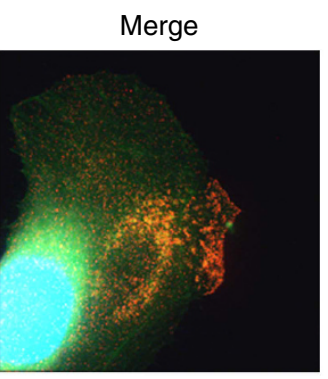

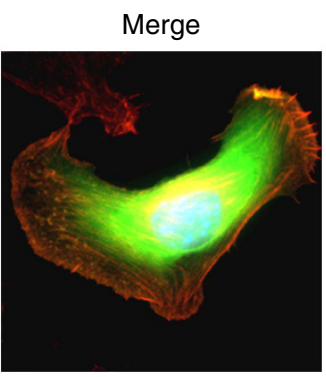

Merge

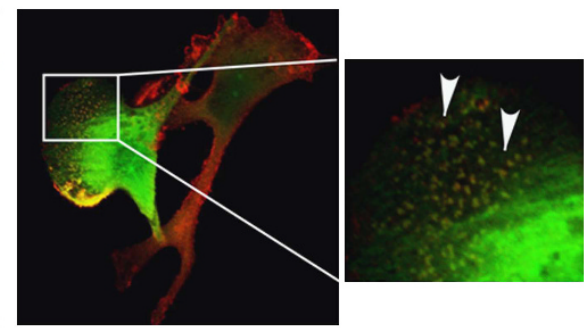

Merge
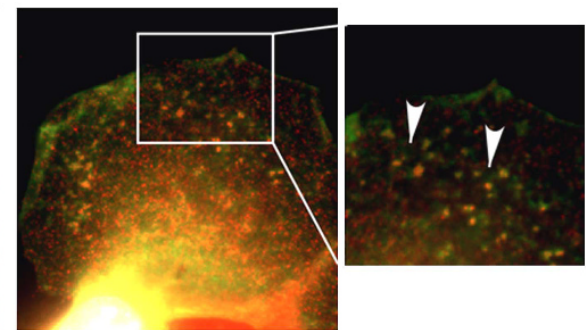

Figure 4. In B16 cells, FAN locates to invadopodia. (A) The B16 F10 cells were stained with phalloidin (green) and cortactin (red), as markers for invadopodia and analysed by fluorescence microscopy. (B) The B16 F10 wt cells were transfected with FAN-GFP and stained for actin (phalloidin, red). (C) The B16 F10 wt cells were transfected with FAN-GFP and stained for cortactin (red). (D) The B16 F10 wt cells were transiently transfected with FAN-GFP and stained for MT1-MMP. Scale bars in microscopy images represent $20 \mu \mathrm{M}$.

role of the TNF-receptor I (TNF-R1) adaptor molecule FAN in mediating motility and invasiveness of B16 melanoma cells, two hallmarks of cancer (Hanahan and Weinberg, 2011). As to the mechanism of FAN action, FAN mediates TNF-induced invadopodia formation by B16 melanoma cells including metalloproteinase activation associated with high proteolytic activity towards the ECM. Thus, FAN regulates migration-associated proteolytic degradation of the ECM, which is a common feature of cancer cells (Wolf and Friedl, 2009).

The role of FAN in tumour cell motility is consistent with previous reports suggesting that FAN is involved in the motility of murine embryonic fibroblasts (Haubert et al, 2007). In addition, FAN regulates the recruitment of neutrophils into the peritoneal cavity (Montfort et al, 2009) and the navigation of leukocytes toward chemotactic cues in zebrafish emanating from tissue damage or infection in vivo (Boecke et al, 2012). Strikingly, our in vivo melanoma mouse model suggested a more complex role of FAN, that is, in evasion, dissemination and invasion of cancer cells, which are important steps in tumour metastasis. At the cellular level, a switch to an invasive phenotype is characterised by the assembly of invadopodia. These actin-rich cellular protrusions serve as secretory sites for ECM-degrading proteases. We have recently reported on the interaction of FAN with $\mathrm{f}$-actin involving the activation of cdc42 (Haubert et al, 2007). F-actin is an integral part of the core structure of invadopodia that also consists of actinassociated proteins including the Arp2/3 complex and cortactin. The core structure is surrounded by a ring of proteins such as vinculin, talin and paxillin (Linder and Aepfelbacher, 2003). As shown in Figure 4, FAN colocalises with two molecules of the core structure of invadopodia, that is, with the f-actin marker phalloidin and with cortactin, suggesting that FAN is involved in invadopodia formation. Moreover, FAN is shown to be essentially required for 

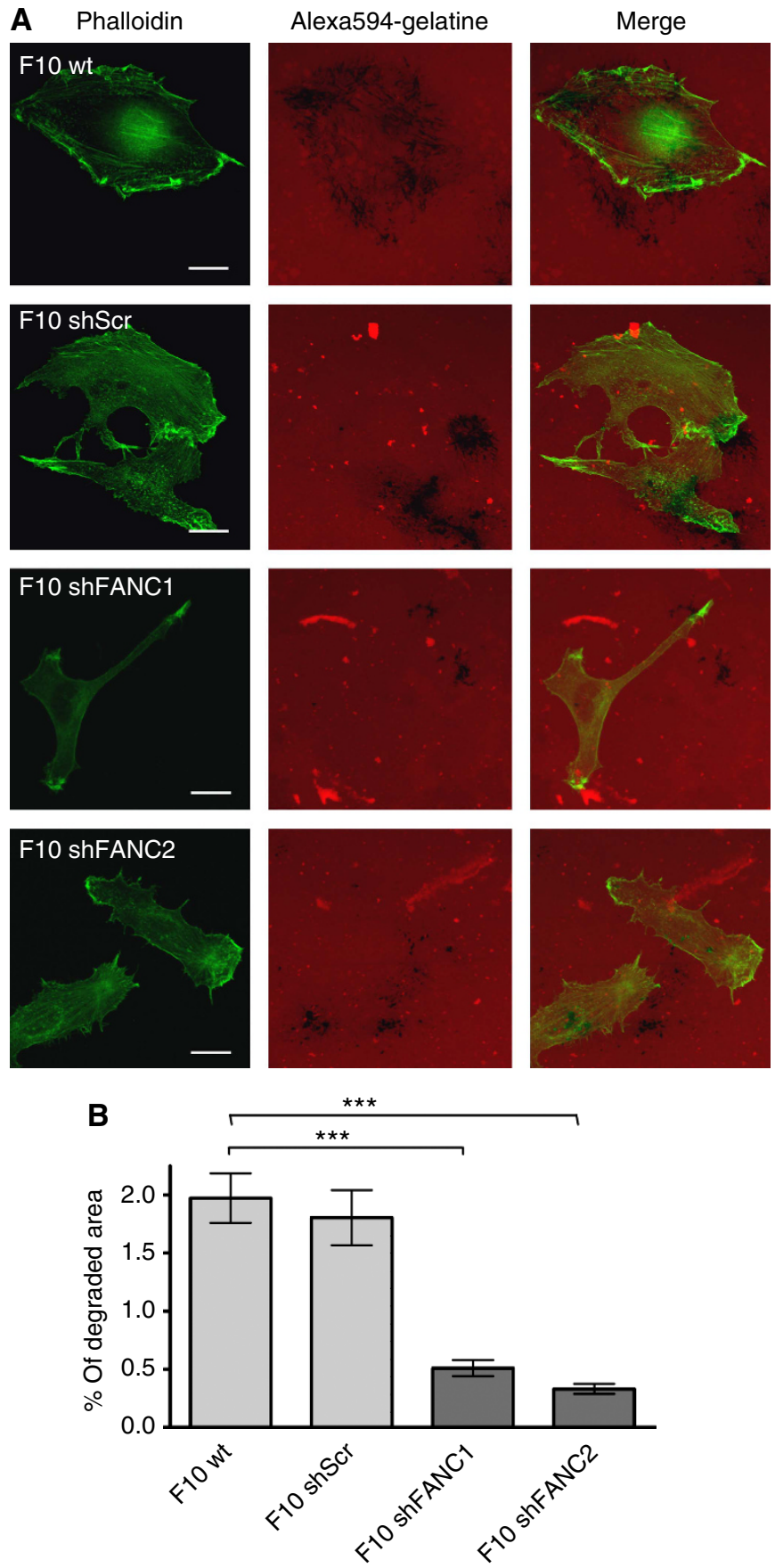

Figure 5. The degradation of ECM is reduced in FAN-deficient B16 cells. (A) Degradation assay of B16 F10 mouse melanoma cells. Degraded matrix imposes as black areas in the red fluorescent background. Green = Phalloidin, red=Alexa594-gelatine. Scale bars in microscopy pictures represent $20 \mu \mathrm{m}$. (B) Quantification of the matrix degradation in B16 F10 mouse melanoma cell lines. ( $n=200$ per cell line). ImageJ was used as a software for quantification. Results are expressed as mean \pm s.e.m. Statistical analyses were standard twotailed Student's $t$-test for two data sets using Prism (GraphPad Inc.). The $P$-values of $\left.<0.05\left(^{(}\right),<0.01{ }^{(* *}\right)$ and $<0.001\left(^{(* *)}\right.$ were deemed as significant, highly significant, and most significant, respectively.

TNF-induced paxillin phosphorylation. Paxillin phosphorylation is functionally involved in the spatiotemporal organisation of invadopodia (Badowski et al, 2008) and linked to the regulation of cellular motility and focal complex turnover (Petit et al, 2000;
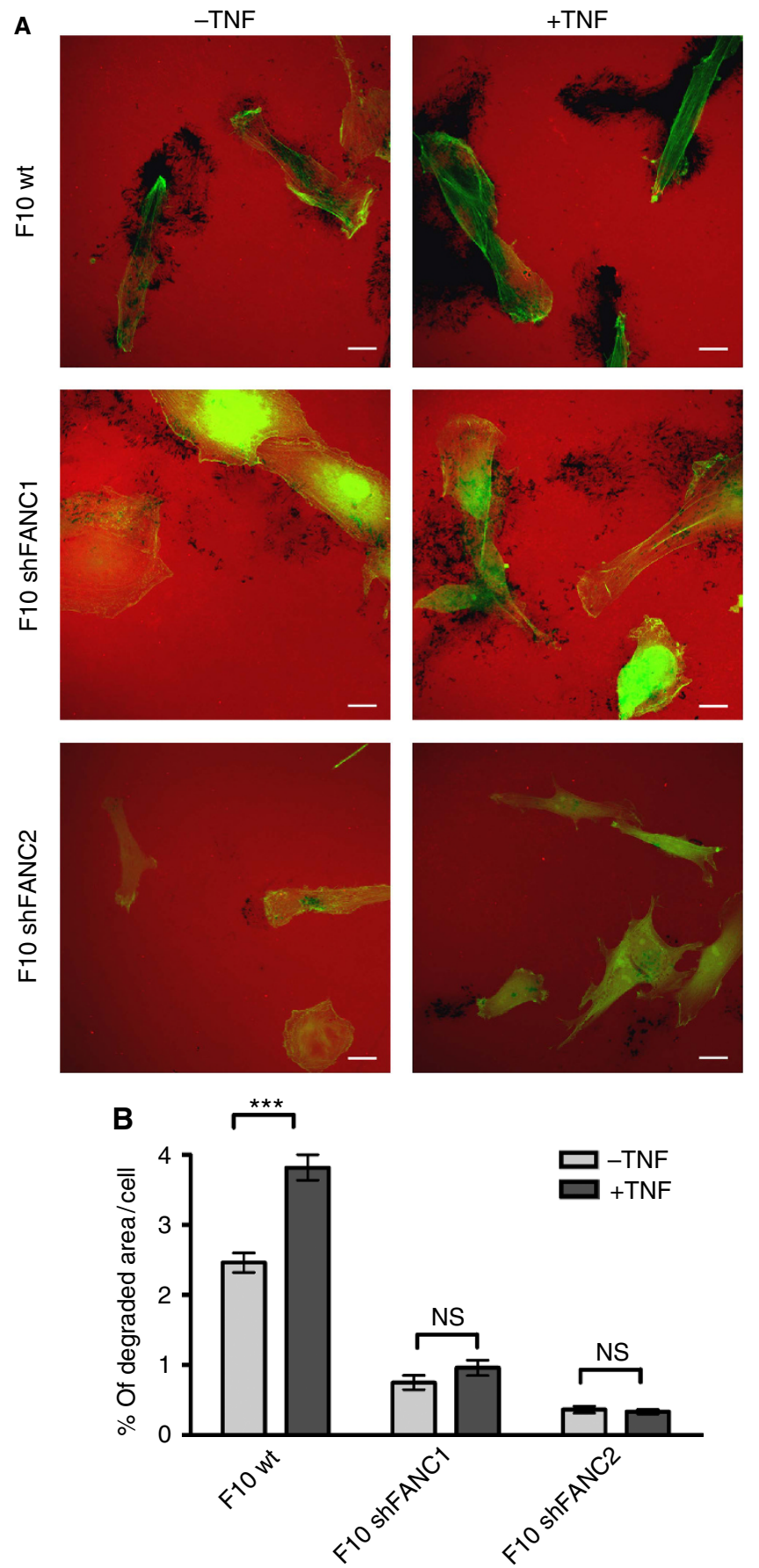

Figure 6. Tumor necrosis factor (TNF)-induced matrix degradation is reduced in FAN-deficient B16 cells. (A) Degradation assay of B16 F10 mouse melanoma cells stimulated with TNF. Degraded matrix is visible as black areas in the red fluorescent background. Scale bars in microscopy images represent $20 \mu \mathrm{m}$. Green = Phalloidin. (B) Matrix degradation by B16 F10 mouse melanoma cell lines in the presence and absence of TNF was assessed by evaluating 200 cells per cell line; ImageJ was used as a software for quantification. Results are expressed as mean \pm s.e.m. Statistical analyses were standard two-tailed Student's t-test for two data sets using Prism (GraphPad Inc.). The P-values of $\left.<0.05{ }^{(*)},<0.01{ }^{(\star *}\right)$ and $<0.001_{(* \star}^{(*)}$ were deemed as significant, highly significant, and most significant, respectively.

Tsubouchi et al, 2002), which is inducible by TNF (Fuortes et al, 1994; Hanna et al, 2001; Brown and Turner, 2004). Thus, our data suggest that FAN through mediating TNF-induced 

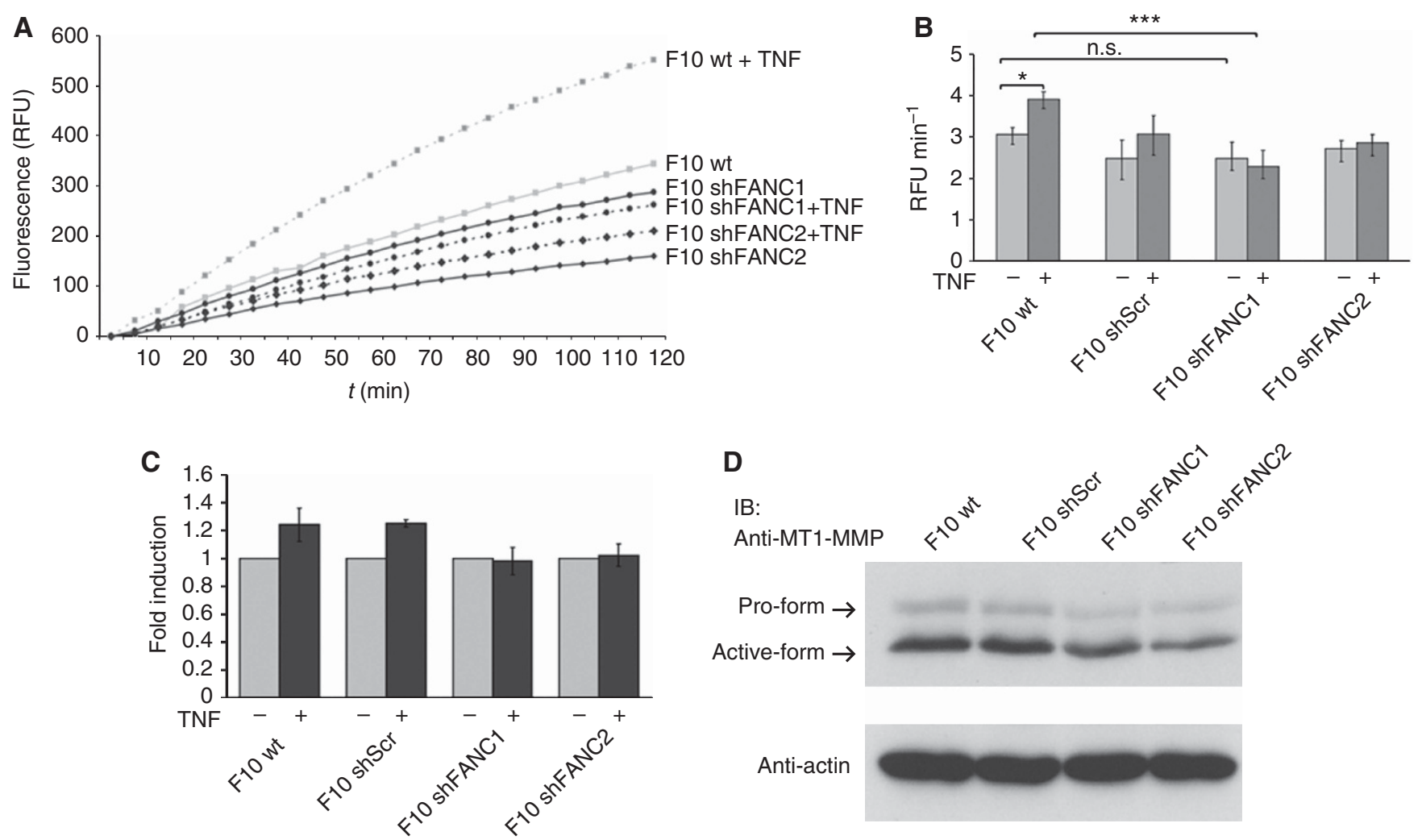

Figure 7. The TNF-induced metalloproteinase activation is mediated by FAN. (A) One representative assay of MMP activation. Active MMPs cleave OmniMMP RED fluorogenic substrate (Enzo) emitting a fluorescent signal. The B16 F10 cells were incubated with the substrate. Increasing fluorescence is measured in RFUs over the course of $2 \mathrm{~h}$. (B) The increase of fluorescence was determined as RFU min ${ }^{-1}$ and used as quantification of enzyme activity. (C) To visualise the induction, the stimulated TNF activity (RFU $\min ^{-1}$ ) was normalised to the unstimulated value. Bars show mean values of three individual experiments, each performed in triplicate. Results are expressed as mean \pm s.e.m. (D) Cell lysates from B16 F10 melanoma cells were prepared, separated by SDS-PAGE and analysed by western blotting using an anti-MT1-MMP antibody. Actin probing ensured equal loading. Statistical

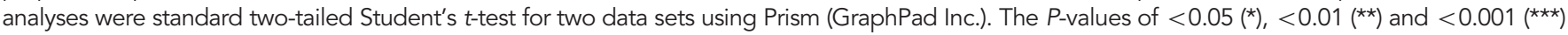
were deemed as significant, highly significant, and most significant, respectively.

phosphorylation of paxillin is involved in the dynamics of invadopodia formation and disassembly.

The downstream pathways by which FAN relays TNF-induced paxillin phosphorylation remain to be determined. In this respect, it is important to note that FAN does not bind to the death domain (DD) of the TNF-R1 (Adam et al, 1996) and stimulates DD-independent signalling pathways and cellular responses. The plethora of TNF-R1 signalling pathways emanate from its intracellular DD. The TNF-R1-associated DD binding protein, TRADD, acts as a platform for the recruitment of defined signalling proteins mediating the canonical TNF-induced activation pathways for caspases and NF- $\kappa \mathrm{B}$ (reviewed by Wilson et al, 2009 ). In contrast, only few downstream targets of FAN have been identified, including RACK1 (Tcherkasowa et al, 2002) and the Polycomb group protein EED (embryonic ectodermal development; Philipp et al, 2010), both of which do not interfere with the caspase and NF- $\kappa \mathrm{B}$ activation pathways. With regard to phosphorylation of paxillin and activation of metalloproteases in invadopodia, the direct interaction partners of FAN remain to be determined, yet may well be found in the recently identified podosome/invadopodia proteome (Cervero et al, 2012).

The signature property of invadopodia is their ability to contact with and degrade the ECM, which is a key function of invasive cancer cells. The results of our study show that FAN-deficient B16 melanoma cells are markedly impaired in their ability to degrade gelatine and to activate metalloproteases. Notably, TNF enhanced the ECM-degrading activity and metalloprotease activity in wt B16 melanoma cells, which is consistent with previous reports demonstrating regulation of monocyte MMP-9 activity by TNF (Leber and Balkwill, 1998). In contrast, FAN-deficient melanoma cells did not respond to TNF stimulation, indicating that FAN is essentially required for both spontaneous and TNF-induced ECM-degrading activity of B16 melanoma cells.

The results of our study suggest the involvement of FAN in the intricate network of proteins regulating the spatiotemporal formation, turnover and function of invadopodia, which seems instrumental for tumour cell migration and metastasis. As FAN also mediates TNF induction of interleukin 6, chemokines and chemokine receptors (Montfort et al, 2009), its role in tumour cell invasiveness might not be restricted to its function in invadopodia dynamics. This places FAN into a special position with respect to the paradoxical role of TNF in cancer. Tumor necrosis factor, while inducing destruction of blood vessels and cell-mediated killing of certain tumours, also acts as a tumour promoter (Balkwill et al, 2005). A dichotomy is emerging where a large part of the tumourpromoting activity of TNF is mediated by FAN, whereas its antitumoural activity is mediated by the TNF-R1 death domaindependent caspase activation pathways. Understanding the intricate role of FAN in the process of TNF-associated tumour promotion will assist in the development of novel cancer therapies. Hwoever, in light of the fact that FAN is also required for the migration of leukocytes (Montfort et al, 2009; Boecke et al, 2012), its possible anti- or pro-tumoural function in the tumour microenvironment remains to be determined. 


\section{ACKNOWLEDGEMENTS}

We thank Anne Buning and Ulrike Karow for expert technical help and Benjamin Yazdanpanah for providing the modified cloning vectors. This work was supported by a grant from the Deutsche Forschungsgemeinschaft, SFB 832 (to MK, OU and HK).

\section{REFERENCES}

Adam-Klages S, Adam D, Wiegmann K, Struve S, Kolanus W, SchneiderMergener J, Krönke M (1996) FAN, a novel WD-repeat protein, couples the p55 TNF-receptor to neutral sphingomyelinase. Cell 86: 937-947.

Adam D, Wiegmann K, Adam-Klages S, Ruff A, Krönke M (1996) A novel cytoplasmic domain of the p55 tumor necrosis factor receptor initiates the neutral sphingomyelinase pathway. J Biol Chem 271: 14617-14622.

Artym VV, Zhang Y, Seillier-Moiseiwitsch F, Yamada KM, Mueller SC (2006) Dynamic interactions of cortactin and membrane type 1 matrix metalloproteinase at invadopodia: defining the stages of invadopodia formation and function. Cancer Res 66: 3034-3043.

Badowski C, Pawlak G, Grichine A, Chabadel A, Oddou C, Jurdic P, Pfaff M, Albiges-Rizo C, Block MR (2008) Paxillin phosphorylation controls invadopodia/podosomes spatiotemporal organization. Mol Biol Cell 19: 633-645.

Balkwill F (2009) Tumour necrosis factor and cancer. Nat Rev Cancer 9: 361-371.

Balkwill F, Charles KA, Mantovani A (2005) Smoldering and polarized inflammation in the initiation and promotion of malignant disease. Cancer Cell 7: 211-217.

Bauvois B (2004) Transmembrane proteases in cell growth and invasion: new contributors to angiogenesis? Oncogene 23: 317-329.

Bingle L, Brown NJ, Lewis CE (2002) The role of tumour-associated macrophages in tumour progression: implications for new anticancer therapies. J Pathol 196: 254-265.

Boecke A, Sieger D, Neacsu CD, Kashkar H, Krönke M (2012) Factor associated with neutral sphingomyelinase activity mediates navigational capacity of leukocytes responding to wounds and infection: live imaging studies in zebrafish larvae. J Immunol 189: 1559-1566.

Bowden ET, Barth M, Thomas D, Glazer RI, Mueller SC (1999) An invasionrelated complex of cortactin, paxillin and PKCmu associates with invadopodia at sites of extracellular matrix degradation. Oncogene 18: 4440-4449.

Brown MC, Turner CE (2004) Paxillin: adapting to change. Physiol Rev 84: 1315-1339.

Cervero P, Himmel M, Krüger M, Linder S (2012) Proteomic analysis of podosome fractions from macrophages reveals similarities to spreading initiation centres. Eur J Cell Biol 91: 908-922.

Chang C, Werb Z (2001) The many faces of metalloproteases: cell growth, invasion, angiogenesis and metastasis. Trends Cell Biol 11: S37-S43.

Clark EA, Golub TR, Lander ES, Hynes RO (2000) Genomic analysis of metastasis reveals an essential role for RhoC. Nature 406: 532-535.

Condeelis J, Pollard JW (2006) Macrophages: obligate partners for tumor cell migration, invasion, and metastasis. Cell 124: 263-266.

Dekker SK, Vink J, Vermeer BJ, Bruijn JA, Mihm Jr. MC, Byers HR (1994) Differential effects of interleukin 1-alpha (IL-1 alpha) or tumor necrosis factor-alpha (TNF-alpha) on motility of human melanoma cell lines on fibronectin. J Invest Dermatol 102: 898-905.

Fuortes M, Jin WW, Nathan C (1994) Beta 2 integrin-dependent tyrosine phosphorylation of paxillin in human neutrophils treated with tumor necrosis factor. J Cell Biol 127: 1477-1483.

Hanahan D, Weinberg RA (2011) Hallmarks of cancer: the next generation. Cell 144: 646-674.

Hanna AN, Berthiaume LG, Kikuchi Y, Begg D, Bourgoin S, Brindley DN (2001) Tumor necrosis factor-alpha induces stress fiber formation through ceramide production: role of sphingosine kinase. Mol Biol Cell 12: 3618-3630.

Haubert D, Gharib N, Rivero F, Wiegmann K, Hösel M, Krönke M, Kashkar H (2007) PtdIns(4,5)P-restricted plasma membrane localization of FAN is involved in TNF-induced actin reorganization. EMBO J 26: 3308-3321.

Hou CH, Yang RS, Hou SM, Tang CH (2010) TNF-alpha increases alphavbeta3 integrin expression and migration in human chondrosarcoma cells. J Cell Physiol 226: 792-799.
Kreder D, Krut O, Adam-Klages S, Wiegmann K, Scherer G, Plitz T, Jensen JM, Proksch E, Steinmann J, Pfeffer K, Krönke M (1999) Impaired neutral sphingomyelinase activation and cutaneous barrier repair in FAN-deficient mice. EMBO J 18: 2472-2479.

Lai FP, Szczodrak M, Block J, Faix J, Breitsprecher D, Mannherz HG, Stradal TE, Dunn GA, Small JV, Rottner K (2008) Arp2/3 complex interactions and actin network turnover in lamellipodia. EMBO J 27: 982-992.

Leber TM, Balkwill FR (1998) Regulation of monocyte MMP-9 production by TNF-alpha and a tumour-derived soluble factor (MMPSF). Br J Cancer $\mathbf{7 8}$ : 724-732.

Linder S (2007) The matrix corroded: podosomes and invadopodia in extracellular matrix degradation. Trends Cell Biol 17: 107-117.

Linder S, Aepfelbacher M (2003) Podosomes: adhesion hot-spots of invasive cells. Trends Cell Biol 13: 376-385.

Montfort A, de Badts B, Douin-Echinard V, Martin PG, Iacovoni J, Nevoit C, Therville N, Garcia V, Bertrand MA, Bessieres MH, Trombe MC, Levade T, Benoist H, Segui B (2009) FAN stimulates TNF(alpha)-induced gene expression, leukocyte recruitment, and humoral response. J Immunol 183: 5369-5378.

Mueller SC, Chen WT (1991) Cellular invasion into matrix beads: localization of beta 1 integrins and fibronectin to the invadopodia. J Cell Sci 99(Pt 2): 213-225.

Murphy DA, Courtneidge SA (2011) The 'ins' and 'outs' of podosomes and invadopodia: characteristics, formation and function. Nat Rev Mol Cell Biol 12: 413-426.

Nakahara H, Howard L, Thompson EW, Sato H, Seiki M, Yeh Y, Chen WT (1997) Transmembrane/cytoplasmic domain-mediated membrane type 1-matrix metalloprotease docking to invadopodia is required for cell invasion. Proc Natl Acad Sci USA 94: 7959-7964.

Peppelenbosch M, Boone E, Jones GE, van Deventer SJ, Haegeman G, Fiers W, Grooten J, Ridley AJ (1999) Multiple signal transduction pathways regulate TNF-induced actin reorganization in macrophages: inhibition of Cdc42-mediated filopodium formation by TNF. J Immunol 162: $837-845$.

Petit V, Boyer B, Lentz D, Turner CE, Thiery JP, Valles AM (2000) Phosphorylation of tyrosine residues 31 and 118 on paxillin regulates cell migration through an association with CRK in NBT-II cells. J Cell Biol 148: 957-970.

Philipp S, Puchert M, Adam-Klages S, Tchikov V, Winoto-Morbach S, Mathieu S, Deerberg A, Kolker L, Marchesini N, Kabelitz D, Hannun YA, Schutze S, Adam D (2010) The Polycomb group protein EED couples TNF receptor 1 to neutral sphingomyelinase. Proc Natl Acad Sci USA 107: $1112-1117$.

Pollard JW (2004) Tumour-educated macrophages promote tumour progression and metastasis. Nat Rev Cancer 4: 71-78.

Pongratz C, Yazdanpanah B, Kashkar H, Lehmann MJ, Krausslich HG, Krönke M (2010) Selection of potent non-toxic inhibitory sequences from a randomized HIV-1 specific lentiviral short hairpin RNA library. PLoS One 5: e13172.

Ridley AJ, Schwartz MA, Burridge K, Firtel RA, Ginsberg MH, Borisy G, Parsons JT, Horwitz AR (2003) Cell migration: integrating signals from front to back. Science 302: 1704-1709.

Rosen EM, Goldberg ID, Liu D, Setter E, Donovan MA, Bhargava M, Reiss M, Kacinski BM (1991) Tumor necrosis factor stimulates epithelial tumor cell motility. Cancer Res 51: 5315-5321.

Tcherkasowa AE, Adam-Klages S, Kruse ML, Wiegmann K, Mathieu S, Kolanus W, Krönke M, Adam D (2002) Interaction with factor associated with neutral sphingomyelinase activation, a WD motifcontaining protein, identifies receptor for activated C-kinase 1 as a novel component of the signaling pathways of the p55 TNF receptor J Immunol 169: 5161-5170.

Tsubouchi A, Sakakura J, Yagi R, Mazaki Y, Schaefer E, Yano H, Sabe H (2002) Localized suppression of RhoA activity by Tyr31/118phosphorylated paxillin in cell adhesion and migration. J Cell Biol 159: 673-683.

Wilson NS, Dixit V, Ashkenazi A (2009) Death receptor signal transducers: nodes of coordination in immune signaling networks. Nat Immunol 10: 348-355.

Wolf K, Friedl P (2009) Mapping proteolytic cancer cell-extracellular matrix interfaces. Clin Exp Metastasis 26: 289-298.

Workman P, Aboagye EO, Balkwill F, Balmain A, Bruder G, Chaplin DJ, Double JA, Everitt J, Farningham DA, Glennie MJ, Kelland LR, Robinson V, Stratford IJ, Tozer GM, Watson S, Wedge SR, Eccles SA 
(2010) Guidelines for the welfare and use of animals in cancer research. Br J Cancer 102: 1555-1577.

Yamaguchi H, Condeelis J (2007) Regulation of the actin cytoskeleton in cancer cell migration and invasion. Biochim Biophys Acta 1773: 642-652.

Yamaguchi H, Pixley F, Condeelis J (2006) Invadopodia and podosomes in tumor invasion. Eur J Cell Biol 85: 213-218.

Zhu N, Lalla R, Eves P, Brown TL, King A, Kemp EH, Haycock JW, MacNeil S (2004) Melanoma cell migration is upregulated by tumour necrosis factor-alpha and suppressed by alpha-melanocyte-stimulating hormone. Br J Cancer 90: 1457-1463.

This work is published under the standard license to publish agreement. After 12 months the work will become freely available and the license terms will switch to a Creative Commons AttributionNonCommercial-Share Alike 3.0 Unported License.

Supplementary Information accompanies this paper on British Journal of Cancer website (http://www.nature.com/bjc) 